\title{
A longitudinal investigation of maternal influences on the development of child hostile attributions and aggression
}

Article

Accepted Version

Healy, S. J., Murray, L., Cooper, P. J., Hughes, C. and Halligan, S. L. (2013) A longitudinal investigation of maternal influences on the development of child hostile attributions and aggression. Journal of Clinical Child \& Adolescent Psychology, 44 (1). pp. 80-92. ISSN 1537-4424 doi:

https://doi.org/10.1080/15374416.2013.850698 Available at https://centaur.reading.ac.uk/36021/

It is advisable to refer to the publisher's version if you intend to cite from the work. See Guidance on citing.

Published version at: http://www.tandfonline.com/doi/full/10.1080/15374416.2013.850698

To link to this article DOI: http://dx.doi.org/10.1080/15374416.2013.850698

Publisher: Taylor \& Francis for the Society of Clinical Child \& Adolescent Psychology

All outputs in CentAUR are protected by Intellectual Property Rights law, including copyright law. Copyright and IPR is retained by the creators or other copyright holders. Terms and conditions for use of this material are defined in the End User Agreement. 


\section{CentAUR}

Central Archive at the University of Reading

Reading's research outputs online 


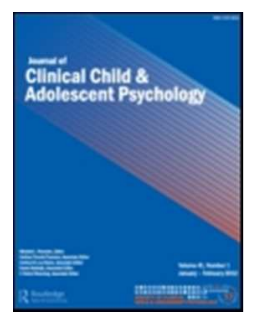

A longitudinal investigation of maternal influences on the development of child hostile attributions and aggression

\begin{tabular}{|r|l|}
\hline Journal: & Journal of Clinical Child and Adolescent Psychology \\
\hline Manuscript ID: & JCCAP-2013-0040.R2 \\
\hline Manuscript Type: & Empirical Article \\
\hline Keywords: & Aggression, Parenting, child, longitudinal, hostile attributions \\
\hline \multicolumn{2}{|l}{} \\
\hline
\end{tabular}

SCHOLARONE ${ }^{m}$

Manuscripts

URL: http://mc.manuscriptcentral.com/jccap Email: mitch.prinstein@unc.edu 


\section{A longitudinal investigation of maternal influences on the development of child hostile} attributions and aggression 


\begin{abstract}
Objective: Aggression in children is associated with an enhanced tendency to attribute hostile intentions to others. However, limited information is available regarding the factors that contribute to the development of such hostile attribution tendencies. We examined factors that contribute to individual differences in child hostile attributions and aggression, focusing on potential pathways from maternal hostile attributions via negative parenting behavior.

Method: We conducted a longitudinal study of 98 mothers and children (47 male, 49 female), recruited from groups experiencing high and low levels of psychosocial adversity. Maternal hostile attributions, observed parenting, and child behaviour were assessed at 18-months and 5-years child age; and child hostile attributions were also examined at 5-years. Independent assessments of maternal and child processes were utilized as far as possible.

Results: Analyses provided support for a direct influence of maternal hostile attributions on the development of child hostile attributions and aggressive behaviour. Maternal hostile attributions were also associated with negative parenting behaviour, which in turn influenced child adjustment. Even taking account of possible parenting influences and pre-existing child difficulties, hostile attributions in the mother showed a direct link with child aggression at 5years. Maternal hostile attributions were themselves related to psychosocial adversity.

Conclusions: Maternal hostile attributions are prevalent in high risk samples, and are related to less optimal parenting behaviour, child hostile attributions, and child aggression. Targeting hostile maternal cognitions may be a useful adjunct to parenting programs.
\end{abstract}

Keywords

hostile attribution bias; aggression; child; parenting; maternal attributions; longitudinal 


\section{A longitudinal investigation of maternal influences on the development of child hostile} attributions and aggression

Cognitive accounts of aggressive behaviour in children and adolescents highlight systematic disturbances in social cognitions. The social information processing model (SIP) (Dodge, 1986; Crick \& Dodge, 1996) outlines six sequential steps in the generation of behavioural responses: the encoding of social cues, cue interpretation, the clarification of goals, behavioural response generation, response selection, and behavioural enactment. Aggressive behaviour is associated with disturbances at each stage of this model, but in particular with biased cue interpretation: aggressive children and adolescents show a bias towards attributing hostile intent to others in ambiguous situations. Although a meta-analytic review of this field has confirmed a robust association between hostile attribution tendencies and externalising difficulties in children (Orobio de Castro, Veerman, Koops, \& Bosch, 2002), the origins of biased SIP are relatively poorly delineated. Social influences, particularly parental attitudes and behaviours, are likely to be important.

\section{Parenting and hostile attribution biases in children}

Poor parenting is implicated in the development of child externalizing problems and is also likely to contribute to hostile attribution tendencies. Individuals are assumed to interpret new social situations by reference to a database of prior experiences, and the schemas they have developed based on those experiences (MacKinnon-Lewis, Lamb, Hattie, \& Baradaran, 1999; Fivush \& Nelson, 2004). Costanzo and Dix (1983) propose that children form attributions about the world, not through reasoning and logic, but by observing and experiencing their parents' behaviour. As such, children who experience harsh or punitive parenting may come to see the world as hostile, with the corresponding schema generalising across contexts.

Previous research provides tentative support for the proposal that parenting behaviours influence child SIP. In a study of 5-6-year old children, parental reports of harsh discipline were linked to SIP disturbances, although the particular association with child hostile attributions 
received only partial support (Weiss, Dodge, Bates, \& Pettit, 1992). In a second study of older children (9-10 years) self-reported paternal psychological control correlated with hostile attributions in boys in instrumental provocation contexts, with equivalent effects in relational contexts that approached significance. However, these findings did not emerge in girls, and maternal self-reported parenting was not related to child hostile attributions in boys or girls (Nelson \& Coyne, 2009). By contrast, a longitudinal study of 9-10 year old aggressive boys found that child perceptions of maternal control and support were associated positively and negatively, respectively, with hostile attributions 1-year later; and child SIP disturbances mediated the association between perceived parenting and aggression (Gomez, Gomez, DeMello, \& Tallent, 2001). Conclusions from these studies are limited by their reliance on parental or child report of parenting behaviour, as both may be subject to bias.

Nelson and Coyne (2008) assessed the relationship between parenting practices, measured via self-report questionnaires, and children's relational intent attributions in a sample of 242 children and their parents. They found aversive parenting by fathers to be associated with a greater tendency toward hostile attributional bias in children, with no significant findings in relation to maternal parenting. Specifically there was a negative correlation with warm and responsive paternal parenting and children's hostile attribution bias and a positive relationship between paternal use of corporal punishment and attribution biases (but only for girls). There was also a correlation between paternal psychological control and children's hostile attribution biases, but this was only found for boys.

\section{Parental attribution biases}

Researchers have also examined parents' own attributional biases, as both a potential influence on parenting style and as a direct contributor to children's attributional tendencies. With respect to the former, parental hostile attribution biases may increase the likelihood of responding to children's problems with harsh discipline practices, which in turn may exacerbate hostile attribution tendencies and externalising symptoms in the child. Previous research has 
demonstrated associations between aggression in children and a maternal tendency to attribute hostile intent to their child (Bickett, Milich, \& Brown, 1996). Moreover, Nix and colleagues (Nix et al., 1999) found that mothers' harsh discipline practices mediated a longitudinal association between maternal hostile attributions about their 4-6 year old children's behaviour and children's later externalising behaviour problems at school. A further longitudinal study found that the combination of maternal hostile attributions in relation to the child and ineffective/irritable discipline predicted an increase in child conduct problems from 5 to 7 -years, suggesting that hostile parental attributions may result in a failure to monitor and adapt to actual child responding (as the parent already 'knows' the child's personality), resulting in reduced flexibility and negative parental behaviour (Snyder, Cramer, Afrank, \& Patterson, 2005). Interestingly, although such research findings suggest a potential pathway from maternal hostile attributions to child aggression that operates via negative parenting and resultant child hostile cognition, this has yet to be examined directly.

Direct associations between parental and child hostile attributions may also exist, as the child observes and internalises their parent's world view. Studies examining the possibility of direct associations between parental and child hostile attributions have yielded mixed results. Two studies of aggressive boys found no association between maternal and child attribution of hostile intent (Bickett et al., 1996; MacKinnon-Lewis, Lamb, Arbuckle, Baradaran, \& Volling, 1992). By contrast, a study of disordered and non-disordered girls and boys demonstrated an association between mothers' and daughters' hostile attribution tendencies. This effect was not found for mothers and sons, suggesting that attributional style could be transmitted along gender lines (MacBrayer, Milich, \& Hundley, 2003). However, Halligan and colleagues (Halligan, Cooper, Healy, \& Murray, 2007) found no direct association between parental and child attributions, even when mother-daughter and father-son associations were considered separately. Conversely, Nelson and colleagues (Nelson, Mitchell, \& Yang, 2008), found that maternal, but not paternal, hostile attributions were related to child hostile attributions, regardless of child gender. 


\section{The current study}

In sum, previous research has demonstrated that parents who interpret their child's behaviour in a hostile way show more negative parenting characteristics (Nix et al., 1999). There is also preliminary evidence for a direct link between parenting behaviour and child hostile attributions (Gomez et al., 2001; Nelson \& Coyne, 2009), as well as partial support for a direct link between parental and child hostile attribution tendencies (Nelson et al., 2008; Halligan et al., 2007). Nonetheless, numerous questions remain. First, although previous research has established that maternal attributions and parenting behaviour are associated both with each other, and with child externalising difficulties, the intervening role of child attributional tendencies has yet to be established. Second, studies examining parental attributions in relation to either parenting behaviour or child outcomes have typically assessed parental attributions specifically in relation to their child. Although, arguably, child-related attributions are the most relevant, as a potentially immediate influence on parenting behaviour, this approach means that children's actual characteristics may confound results and make the direction of effects difficult to ascertain. Third, existing research in the area has typically examined parenting behaviour based on parent or child report, both of which have significant limitations, particularly when parent and/or child hostile attributions are being measured simultaneously. Direct observations of parenting behaviour are required (Zaslow et al., 2006).

In the current study, we conducted a comprehensive examination of associations between maternal parenting behaviour, maternal attributional tendencies, child attributional tendencies, and child aggression, with the aim of testing hypothesised pathways to child aggression that are summarised in Figure 1. To this end, we conducted detailed assessments in a sample of 98 fiveyear old children and their mothers, recruited as part of a longitudinal study of the impact of psychosocial adversity on child development (Cronin, Halligan, \& Murray, 2008; Halligan et al., 2013; Sheridan et al., 2013; Gunning, Halligan, \& Murray, 2013). Almost half the families in the study were characterised by high levels of adversity (including socioeconomic deprivation, lacking 
education/employment, low social support, feelings of anxiety and depression), ensuring a reasonable distribution of child externalising difficulties. Maternal attributions and parenting style were assessed at both 18-months and 5-years; and child hostile attributions and aggression were assessed at the 5-year follow-up. In order to address limitations of the existing literature, at 5-years maternal hostile attributions were assessed in both general and child-specific contexts, with the former allowing an examination of maternal hostile attributions that is unaffected by actual child characteristics; parenting behaviour was measured via direct observation; and child aggression via teacher report. Thus, we generated wholly independent assessments of maternal and child attributions and behaviour. In addition, we assessed both relational and overt components of aggression in children, based on some previous observations that hostile attributions may be differentially related to these aggression domains (Crick \& Dodge, 1996; Mathieson et al., 2011). We examined cross-sectional and longitudinal interrelationships among variables, specifically focusing on potential influences of maternal hostile attributions on both child hostile attributions and aggressive behaviour; and on maternal parenting behaviour.

We took account of psychosocial adversity in our analyses, as it is known to be associated with both child externalising problems (Shaw, Winslow, Owens, \& Hood, 1998; Rutter, Giller, \& Hagell, 1998) and less optimal parenting styles (O'Connor, 2002), including in the current sample (Halligan et al., 2013). In principle, psychosocial adversity might also influence intervening cognitive processes, including child tendency to attribute hostile intent, or maternal attributions relevant to parenting behaviour. Chen and colleagues found elevated rates of hostile interpretations in low SES children and adolescents (Chen \& Matthews, 2001), leading to the proposal that SES serves as a proxy for exposure to adverse interpersonal and community circumstances, which directly informs the development of a hostile world view (Chen, Langer, Raphaelson, \& Matthews, 2004). As the current sample included groups of high and low adversity families, the examination of social context as a distal influence on maternal and child attributions was of particular interest. 


\section{Methods}

Study procedures were approved by the University of Reading and NHS research ethics committees. Mothers provided written informed consent prior to participating in each stage of the study, and were given a small reimbursement for their time (GBP 20).

\section{Recruitment}

We screened primiparous mothers attending their routine 20 -week antenatal scan appointment at a local maternity unit (Royal Berkshire Hospital, Reading, UK) using a 20 item questionnaire indexing level of psycho-social adversity (Cronin et al., 2008). Items assess socioeconomic disadvantage (e.g., low income, poor housing, lack of phone/car, single parenthood/low social support) and associated feelings of distress (e.g., symptoms of depression/anxiety). From the screened sample (73\% of the potential population), we recruited 67 mothers scoring above a cut off defining the $20 \%$ highest scores (high risk group), along with 68 mothers with scores in the $40 \%$ lowest scoring range (low risk group). Cut-offs were based on pilot work with the questionnaire, and reflected our goal of recruiting distinct groups of women for whom the psychosocial context was clearly problematic versus not problematic. Recruitment was via telephone contact and/or a letter, and high versus low risk women did not differ in terms of recruitment percentages (low risk 60\%, high risk 64\%: $\chi^{2}=0.56, \mathrm{df}=1, \mathrm{~ns}$ ). After excluding those delivering prematurely or withdrawing from the study, numbers in high and low risk groups were 58 and 63 respectively. On the questionnaire's principal demographic indices of adversity, high risk women were, compared to the low risk group, younger $[M=19.7, S D=3.3$ vs. $M=30.6, S D=$ 3.3 years; $\left.t_{120},=18.3, P<0.001\right]$, more often single [53.4\%vs $\left.0 \% ; \chi^{2}(1)=45.9, P<0.001\right]$, and unemployed [63.2\% vs3.1\%, $\left.\chi^{2}(1)=50.4, P<0.001\right]$, and fewer were educated beyond 16 years $\left[14.8 \%\right.$ vs. $\left.96.8 \%, \chi^{2}(1)=80.8, P<0.001\right]$. The sample was predominantly white $(86 \%)$, but included a proportion of mixed race (9\%), black (3\%) and Asian (2\%) participants. Ethnic minorities were better represented in the high $(21 \%)$ versus low $(8 \%)$ risk group versus $\left(\chi^{2}=4.23\right.$, $\mathrm{df}=1, p=0.04)$. Proportion of female infants was similar for high $(51.7 \%)$ and low $(51.6 \%)$ risk 
groups. Participants initially completed assessments in the perinatal period, specifically at 28weeks and 34-weeks gestation, and at 10-days, 4-weeks, 7-weeks and 12-weeks postpartum. Follow-ups were completed at 12-months, 18-months and 5-years. Of the 121 families originally recruited, $98(81 \%)$ completed the 5-year assessments. Measures relevant to the current study were obtained at 18-months, when maternal hostile attributions, parenting behaviour and child behavioural problems were examined; and at 5-years, when child hostile attributions and aggressive behaviour, as well as maternal hostile attributions and parenting behaviour, were each assessed.

\section{Child Measures}

Behaviour Screening Questionnaire. At the 18-month assessment mothers completed an age-adapted version of the BSQ (Richman \& Graham, 1971), in semi-structured interview format. The BSQ was originally developed as a screen to identify a range of clinical problems in 3-yearold children. The version used in the current study comprised a reduced set of 8 -items which has previously been found to be appropriate for children in the current study age range (Murray, 1992; Ghodsian, Zajicek, \& Wolkind, 1984). We analyzed scores for items specifically addressing externalizing-type difficulties (temper tantrums and hard-to-manage behaviors). The scale has previously been shown to have external validity (as evidenced by associations with other indices of child behavior problems and discrimination of high versus low risk groups) (Ghodsian, Zajicek, \& Wolkind, 1985; Murray, 1992; Halligan et al., 2013) and acceptable reliability (Ghodsian et al., 1984; Richman \& Graham, 1971). Internal consistency for the externalizing scale used in the current study was acceptable $(\alpha=.78)$.

Teacher reported aggression. At the 5 year assessment, teachers completed the Social Behaviour Scale (SBS) - Teacher form (Crick, Casas, \& Mosher, 1997), rated on 5 point scales (from 1 'never/almost never true' to 5 'always/almost always true'). The SBS includes subscales indexing relational (6 items) and overt (6 items) aggressive traits, which, in combination form a total aggression score. The SBS is a widely used measure with established validity and reliability 
(Crick et al., 1997; Juliano, Werner, \& Wright Cassidy, 2006). Internal consistency in the current study was acceptable for the total aggression score $(\alpha=.89)$, and for relational $(\alpha=.82)$ and physical $(\alpha=.89)$ subscales.

Ambiguous social scenarios. At 5-years, child hostile attributions and aggressive solutions were measured using standard ambiguous scenarios $(\mathrm{n}=8)$, including both relational and overt provocations to ensure that situations relevant to both males and females were presented (Crick, Grotpeter, \& Bigbee, 2002; Dodge, Pettit, Bates, \& Valente, 1995; MacBrayer et al., 2003). An example of a relational scenario is: "Imagine that you are standing in the corridor one morning before school starts. As you are standing there, two children from your class walk by. As they pass by you, they look at you, whisper something to each other, and laugh.” An example of a physical scenario is: "Pretend that you are standing on the playground playing catch with a $<$ boy/girl> named $<$ The Protagonist $>$. You throw the ball to $<$ The Protagonist $>$ and $<$ he/she $>$ catches it. You turn around, and the next thing you realise is that $<$ The Protagonist $>$ has thrown the ball and hit you in the middle of your back. The ball hits you hard, and it hurts a lot."

The scenarios utilised have been widely used in previous studies and responding has been found to distinguish aggressive and non-aggressive children (Crick et al., 2002; Dodge et al., 1995; MacBrayer et al., 2003). Because of the relatively young age of the children tested, doll play was used for the enactment of scenarios. For each scenario, children were asked to imagine themselves in the situation, with dolls/drawings of the background location used to enact each scenario. Following each scenario children were asked "Why do you think $<$ The Protagonist $>$ acted that way". Children enacted their responses using dolls, along with verbal description. We conducted pilot work which established that children of study age were able to engage with the tasks and that there was sufficient variability in responses. A single rater, blind to all other participant information, coded video recordings of child responses, using rules pre-specified in the literature (Dodge, 1980; Crick \& Dodge, 1996; Bickett et al., 1996). A non-hostile attribution (scored 0) was assigned if the negative event was attributed to: an accident, misunderstanding, 
temporary state, third party or circumstance; to some neutral state or characteristic of the subject themselves; or to the protagonist acting in order to try to help or benefit the subject. A hostile attribution (scored 1) was assigned if the negative event was attributed to: an intentional or goaldirected negative action by the protagonist; or to a negative personality trait ascribed to the protagonist that suggests that they acted with hostile attributions, indifference, lack of sensitivity, or lack of concern. Mean scores were derived. A second blind rater scored a randomly selected subset of 14 child assessments (14\%); the intra-class correlation showed a high level of agreement $(r=.98, P<.001)$

\section{Maternal Measures}

Parenting observations. At 18-months mothers and infants were filmed in the course of unstructured play based on three 2.5-minute consecutive sessions with a series of age-appropriate toys (Wolke, Skuse, \& Mathisen, 1990). Maternal insensitive parenting (unresponsive parenting behavior that is low in warmth and acceptance, and fails to take appropriate account of infant cues) was rated on standard 5-point scales for each of the three segments (Murray, FioriCowley, Hooper, $\&$ Cooper, 1996) and an average score for the interaction computed.

At 5-years, dyads completed a 15-20 minute interaction in three sections: first, a 10-minute free play period using Playdoh; second, a 5-minute period during which mothers were asked to put the Playdoh aside and to engage their child in playing with a shape selector toy, appropriate for a much younger child; third, a tidy-up task where mothers were instructed to direct their child in clearing up all toys. In sum, the interaction included a period of normal play, as well as activities likely to provoke conflict between maternal and child goals (Dadds, Mullins, McAllister, \& Atkinson, 2003; Gardner, Sonuga-Barke, \& Sayal, 1999). Video recorded interactions were coded on five dimensions by two raters, blind to other variables: the extent of negative control (coding verbal commands/control), intrusive behaviours (actions that impose a maternal agenda at the expense of child activities), hostile maternal behaviours (threatening, yelling, criticising the child), a lack of engagement with the child, and insensitive/non-contingent responding. Double coding of 
a minimum $10 \%$ of interactions was completed to assess inter-rater reliability at both 18 -months and 5-years. Intraclass correlations ranged from adequate to excellent (.77 to .90).

Parental Concerns and Constraints Interview (PCCI). Mothers were interviewed using the PCCI (Pettit, Bates, \& Dodge, 1997) at the 18-month assessment. Mothers were presented with five hypothetical situations in which their child misbehaved, and were asked to provide a rationale for their child's actions. For example: "Imagine that you and your child are at a playgroup. All the children are playing and running around excitedly. One of the other children bumps into your child who then falls over. The other children laugh. Your child gets upset and then pushes the other child to the ground." Responses were scored for maternal hostile attributions, indexed via the number of maternal interpretations provided for the child's behaviour that blamed negative child traits or characteristics (versus situational or state factors). Coding was completed by two researchers, blind to other scores. Double coding of a randomly selected subset of interviews $(\mathrm{n}=$ $10,10 \%$ of sample) demonstrated acceptable inter-rater reliability $($ kappa $=.79)$. Examination of the resultant set of responses indicated that $72 \%$ of mothers showed no hostile attributions on this measure, therefore responses were dichotomised as hostile attributions present (coded 1) versus absent (0). The PCCI has been previously used in a number of studies, and analyses have supported the validity of this measure (Pettit et al., 1997; Pettit, Laird, Bates, Dodge, \& Criss, 2001), including associations with other indices of maternal responding and child outcomes. In addition, the scenario based approach is typical of that used to index maternal attributions in previous work (MacBrayer et al., 2003; Nix et al., 1999).

Maternal Hostile Attribution Questionnaire (HAQ). At 5-years mothers completed the HAQ, a questionnaire which examined attributions for 16 hypothetical situations, including a number not involving their child. In previous work this measure of maternal hostile attributions distinguished mothers of clinically referred versus non-referred children and was associated with measures of child hostile attribution tendencies (Bickett, Milich, \& Brown, 1996; MacBrayer, et al., 2003). In the current study, mothers were presented with written ambiguous vignettes and 
asked to imagine themselves in that situation. For each vignette, mothers provided: a) a written explanation of why they thought the situation had occurred; and b) a rating of the extent to which they believed that their explanation was correct (from 1 'somewhat' to, to 4 'extremely' likely). The latter was introduced following pilot work in order to capture variation in the strength with which mothers held their beliefs. Six of the vignettes indexed general hostile attribution tendencies (situations described interactions with other adults, friends and colleagues), for example: "Imagine you are seated at a bar in a restaurant. The people next to you are laughing and talking. One of them brushes against you. You do not pay any attention to this. This same person then bumps into you, causing you to spill your drink. You look over at the person and she is laughing." Ten vignettes indexed child-specific attributions, for example: "Imagine that you give your child a "time out" in the living room. When you walk into the room to tell $<$ him/her $>$ that the time out is over, you see that an expensive vase is lying broken on the floor." In order to ensure that scenarios represented a range of relevant experiences, both relational and overt provocations were included (proportion of relational was $60 \%$ for child scenarios, $67 \%$ for general scenarios).

Scenario Responses were coded as hostile (1) or benign (0), rated as per the child assessment. Scores for analyses derived from combining hostile attribution codes and accompanying 'belief' ratings (i.e., hostile attributions scored between 1 and 4, according to the corresponding rating of "correctness"), and mean scores for both the general and child-specific scenarios were derived separately (resultant possible range 0 to 4 ). Coding of responses was completed by a single rater, blind to other variables. A second blind rater coded a sub-sample of 10 questionnaires $(10 \%)$, and inter-rater reliability was established $(\mathrm{kappa}=.79)$.

\section{Data Analyses}

Hypothesized longitudinal models were examined via path analysis, conducted using Maximum Likelihood Estimation using MPLUS 4.2 software (Muthen \& Muthen, 2007). In addition to chi-square tests, model fit was evaluated using the Comparative Fit Index (CFI), the Tucker Lewis Tucker Lewis Index (TLI) and the Root Mean Square Error of Approximation 
(RMSEA). For the CFI and TLI values above .90 indicate acceptable fit, and those above .95 good fit. For the RMSEA values less than 0.08 show acceptable fit and below 0.05 good fit. Prior to the construction of models, correlational analyses were used to screen for potential influences of gender and risk on key outcomes. Gender effects were then included in models as appropriate. Preliminary models were constructed without risk effects, due to both the complexity that resulted from the inclusion of this variable and the focus of research questions. Following the construction of satisfactory models that did not include risk, risk effects were added with the goal of examining both the relevance of risk to maternal and child hostile attributions, and also the impact of this potential confound on existing pathways.

As already noted, 98 of the original 121 families were retained in the study at 5-years. There was greater attrition from the high versus the low-risk group (17/58 versus $6 / 63$ at 5 -years; $\chi^{2}=$ $7.68, \mathrm{df}=1, p=0.006)$. Rates of refusal were similar across groups ( 3 high versus 2 low risk mothers), but we were unable to trace a higher proportion of the high risk families (14 high versus 4 low risk mothers). We used analysis of variance to examine original adversity scores in relation to retention status and risk group, with analyses conducted for both 18-month and 5-year retention status. Retention status showed no significant association with initial adversity scores, either as a main effect $\left(P>.28\right.$, partial $\left.\eta^{2}<.01\right)$, or in interaction with risk group $\left(P>.62\right.$, partial $\left.\eta^{2}<.002\right)$. Within group comparisons yielded the same conclusions. Thus, those who were lost to follow-up appeared to be comparable to those in their group who were retained in the study. Path analyses were based on the 105 participants who had data available for 18-months and/or 5-years, with missing data modelled using full information maximum likelihood estimation, as recommended for minimizing the introduction of bias due to missing data (Allison, 2003).

\section{Results}

\section{Preliminary analyses}

Table 1 presents descriptive statistics and Table 2 presents a full correlation matrix for key study variables. Of the 98 children assessed at 5-years, 47 were male and 51 female. Correlational 
analyses identified gender effects as follows: boys scored higher than girls in terms of teacherrated total aggression on the SBS and also on the physical aggression subscale; and mothers of boys were more insensitive than mothers of girls in their interactions at 18-months and showed a trend towards using more negative control at 5-years.

Examination of the correlation matrix presented in Table 2 yielded some key observations. First, child hostile attributions were not significantly correlated with teacher-reported total child aggression scores on the SBS but were significantly correlated with relational aggression subscale scores (Table 2) ${ }^{1}$. In the light of these findings, we modelled total aggression scores and relational aggression scores separately, with our examination of influences on child hostile attribution scores being restricted to the latter. Second, general (but not child-specific) maternal hostile attributions at 5-years were significantly associated with parenting and child adjustment; our analyses therefore focused exclusively on these general maternal hostile attribution scores. Third, although small in magnitude ${ }^{2}$, the overall pattern of correlations was consistent with hypothesised effects. Thus, maternal and child hostile attributions showed both prospective and concurrent associations. Likewise, maternal hostile attributions were associated with maladaptive parenting behaviour, particularly at the 5-year assessment. Negative parenting, in turn, was significantly correlated with child hostile attributions at 5-years, although there was no equivalent longitudinal association from 18-month maternal insensitivity. Finally, as expected, there were also significant associations between parental behaviour and child aggression at 5-years.

\section{Maternal influences on child overall aggression scores}

We used path analysis to examine cross-sectional and longitudinal pathways from maternal hostile attributions to child total aggression scores at 5-years. Our proposed model included pre-

\footnotetext{
${ }^{1}$ We also tested for domain specificity across relational/overt types of aggression/scenarios. For relational aggression, significant correlation was found with relational scenario hostile attribution scores $(r=.21, P=.041)$ but not with overt attribution scores $(r=.14, P=.27)$; however, correlations were not significantly different in magnitude $(t=0.62$, $P=.54)$. For overt aggression, there were no significant correlations with either relational or overt hostile attributions. (both $r<$. 11).

${ }^{2}$ These effects were similar in magnitude for female and male children. Note that there were no significant effects when scores for relational versus overt scenarios were computed and the possibility of domain specificity in transmission was examined. However, the small number of scenarios indexing relational/overt dimensions limited these analyses.
} 
existing child problem behaviours at 18-months, as well as maternal parenting behaviour (see

Figure 2). Two modifications were made to this proposed model in the light of preliminary analyses. First, correlational analyses provided no evidence for a prospective influence from maternal hostile attributions at 18 -months to negative parenting at 5-years; and the pathway from child behaviour at 18-months to maternal hostile attributions at 5-years was not considered plausible when the focus was exclusively on general versus child specific maternal attributions. These pathways (dashed lines in Figure 2) were excluded. Second, gender effects on maternal parenting behaviour and child aggression were included. The resultant model showed a good fit $\left(\chi^{2}\right.$ $=5.79, \mathrm{df}=7, p=0.56 ; \mathrm{CFI}=1.00, \mathrm{TLI}=1.00 ; \mathrm{RMSEA}<0.01)$, and is depicted in Figure 2 including standardized regression coefficients. Several notable observations emerged from the model. First, there were within time-point associations at both 18-months and 5-years between maternal hostile attributions and less optimal parenting behaviour. These associations were present even once existing levels of child problem behaviours (18-months) or aggression (5-years) were taken into account in the model. Second, between 18-months and 5-years there was modest stability in maternal hostile attribution tendencies, even though the assessment differed substantially in type and content over this period. Third, at 5-years, there was a significant association between maternal general hostile attributions and child aggression, even once any effects via parenting behaviour were taken into account.

\section{Maternal influences on child relational aggression}

We constructed a second model that focused on child relational aggression at 5-years. As relational aggression showed a significant association with child hostile attributions, influences on the latter could also be examined in the context of this model. Given the potential complexity associated with the introduction of an additional variable, and the lack of gender effects on relational aggression or child hostile attributions, gender was not included in the model. In addition, only pathways that were supported by basic correlations were examined. The resultant model showed an adequate fit $\left(\chi^{2}=9.93, \mathrm{df}=7, p=0.19 ; \mathrm{CFI}=0.96, \mathrm{TLI}=0.89 ; \mathrm{RMSEA}=\right.$ 
$0.63)$. The pathway from 18-month parenting to 5-year relational aggression was weak $(r=.10)$; when this was dropped, fit indices were generally consistent with a good fitting model $\left(\chi^{2}=10.7\right.$, $\mathrm{df}=8, p=0.22 ; \mathrm{CFI}=0.97, \mathrm{TLI}=0.91 ; \mathrm{RMSEA}=0.057)$. Figure 3 shows the resultant model's standardised regression weights, with the excluded pathway indicated by a dashed line.

Associations between maternal attributions and both parenting behaviour and child difficulties were supported, as for the model of overall aggression. An intervening role for child hostile attributions was less clear. There was a significant longitudinal pathway from 18-month maternal hostile attributions to 5-year child hostile attributions. However, regression weights for pathways from 5-year maternal hostile attributions and negative parenting behaviour to child attributions were small in magnitude and not statistically significant; and the association between child hostile attributions and relational aggression was similarly modest.

\section{Psychosocial adversity effects}

Our sample included groups distinguished by high versus low levels of psychosocial adversity, and the correlational analyses presented in Table 2 identified several associations between adversity status and mother/child outcomes. Notably, there were significant links with maternal hostile attribution indices: at 18 -months, $53.3 \%$ of high versus $18.3 \%$ of low risk mothers provided hostile interpretations on the PCCI for their child's behaviour, and at 5-years significantly higher general maternal hostile attribution scores were present in the high $(M=1.09$, $S D=0.64)$ versus low $(M=0.58, S D=0.48)$ adversity group. As highlighted in previous reports with the same sample (Halligan et al., 2013), relative to their low adversity counterparts, mothers in the high adversity group also showed less optimal parenting behaviours at 18-months and 5years, and children showed more problem behaviours at 18-months (see Table 2). These four associations were modelled in path analyses, particularly with the aim of examining the impact on pathways already identified in previous models.

With regards to the model examining total aggression scores as outcome, adding effects of adversity status resulted in a model which showed a reasonable fit $\left(\chi^{2}=12.3, \mathrm{df}=9, p=0.20\right.$; CFI 
$=0.97, \mathrm{TLI}=0.94 ; \mathrm{RMSEA}=0.059)$. Regression coefficients for pathways between adversity group status and maternal attributions were robust $(18$-months estimate $=1.00, \mathrm{SE}=0.27$, standardised coefficient $=.45 ; 5$-years estimate $=0.47, \mathrm{SE}=0.14$, std coeff. $=.39$ ). Two previously identified pathways were weak $(r<.10)$ when risk was included. Specifically the association between child behavioural difficulties (BSQ) and maternal insensitive parenting at 18months $($ estimate $=0.001, \mathrm{SE}=0.13$, std coeff. $=.001 . \mathrm{ns})$, and that between maternal 18 -month and 5 -year hostile attributions (estimate $=0.02, \mathrm{SE}=0.06$, std coeff. $=.03, \mathrm{~ns}$ ), showed essentially zero effects. Eliminating these two pathways resulted in a good fitting model $\left(\chi^{2}=10.7, \mathrm{df}=10, p\right.$ $=0.38 ; \mathrm{CFI}=0.99, \mathrm{TLI}=0.99 ; \mathrm{RMSEA}=0.026)$. Equivalent analyses including risk indices in the modelling of relational aggression yielded identical conclusions, with the same three existing pathways being weak following the inclusion of risk.

\section{Discussion}

We examined several possible pathways to child aggression from maternal attributions, generating the following findings. First, our analyses provided tentative support for a direct association between maternal and child hostile attribution tendencies. In particular, we found a longitudinal link between maternal hostile attributions at 18-months and child attributions at 5years was maintained even once possible indirect influences via parenting behaviour were included in our model. This observation extends previous reports of cross-sectional links between maternal and child attributions, and suggests that maternal hostile attributions may be a sustained influence on children's social understanding. Second, we found associations between maternal hostile attributions and less optimal parenting behaviour, highlighting the potential for maternal hostile attributions to also influence child outcomes indirectly, via negative parenting behaviour. Third, general maternal hostile attributions showed direct, cross-sectional associations with child aggression at 5-years, even once any effects via parenting behaviour were taken account of, highlighting the importance of taking account of maternal social cognition. Fourth, we found limited support for the putative role of child hostile attributions in contributing to associations 
between maternal hostile attributions/parenting and child aggression. Fifth, psychosocial adversity was an important correlate of maternal (but not child) hostile attribution tendencies, as well as of parenting behaviour (Halligan et al., 2013). The inclusion of longitudinal measures, the simultaneous modelling of several sources of potential maternal influence, the direct observation of parenting, and the independence of measures at 5-years represent methodological advantages relative to previous work in the field.

\section{Direct associations between maternal and child hostile attributions}

In support of a developmental influence of maternal hostile attributions on child aggressogenic cognition, we found a prospective, direct association between 18-month maternal and 5-year child hostile attributions. Thus, longitudinal nature of this association is significant, as it supports the assumption that child may observe and internalize the hostile interpretative style of their parents over time. At the same time, we failed to establish a robust link between maternal and child attributions at 5-years. Although this null finding must be interpreted cautiously in the light of the sample size, our observations echo similarly mixed results across previous studies, in which direct parent-child correlations in hostile attributions have proved difficult to establish. Thus, in addition to numerous null findings (Halligan et al., 2007; Bickett et al., 1996; MacKinnon-Lewis et al., 1999), where positive associations have been identified they have typically been limited to a subset of the sample studied and of the indices of hostile attributions obtained (MacBrayer et al., 2003; Nelson et al., 2008). Variations in the approach taken to the measurement of parental attributions may have contributed to mixed findings, including in the current study. Nonetheless, the weight of evidence suggests that maternal hostile attribution tendencies are likely to exert relatively modest direct influences on child hostile social cognition.

\section{Maternal hostile attributions as an influence on parenting behaviour and child aggression}

Our findings identified potential influences of maternal hostile attribution tendencies on parenting behaviour - specifically, insensitivity in 18-month interactions and the use of negative control. These findings are in line with previous reports of associations between maternal hostile 
attributions and child aggression (Bickett et al., 1996; Nix et al., 1999). In particular, in their 4year longitudinal study, Nix and colleagues (1999) found that harsh discipline practices mediated a predictive association between mother's hostile attribution tendencies and children's later externalizing problems. Notably, previous research has typically focused on maternal hostile attributions occurring in relation to their own child's behaviour. Such a focus is warranted, as child specific attributional tendencies are the most likely to influence maternal parenting behaviour. However, conclusions in this context are complicated by the likely reciprocal relationship between children's externalising symptoms and variation in mothers' proclivity to assume the worst of them. In the current study, in addition to concurrent associations between maternal hostile attributions made in relation to the child and child difficulties at 18 -months, we also found that mothers' general hostile attributions (indexed by interpretations of situations involving other adults) at 5-years were associated with both negative parenting and child externalising symptoms. This finding is significant, as the assessment used in this case represents a measure of maternal hostile attribution tendencies that is not contaminated by existing representations of/experiences with a specific individual (i.e., her child). The fact that this association held even once pre-existing child behavioural problems at 18-months were included in the model also suggests that maternal attributions are not only a reflection of the presence of child behavioural problems. Unexpectedly, maternal hostile attributions in relation to the child at 5-years were unrelated to negative parenting and other outcomes. We used open-ended vignettes to assess maternal attributions versus the rating scale approach utilised in previous research (Nix et al., 1999), and mothers may have had reservations about expressing overtly negative views of their child, particularly in this non-clinical sample. However, it may also be that our general assessment of attributions at 5-years tapped into a more fundamental, maternal characteristic, explaining the stronger link to parenting behaviours.

A direct association between maternal hostile attributions and child aggression still held when our models took parenting characteristics into account. There are several possible explanations for this link. Maternal hostile attributions may influence aspects of parental 
behaviour or the parent-child relationship that are not necessarily captured by laboratory

observations, particularly the tendency to engage in harsh or over-reactive discipline in the context of perceived child transgressions. Further exploration of the contexts in which maternal attributions are particularly activated may be fruitful in the development of related interventions. Maternal interpretations of child behaviour may also be conveyed to a co-parent or to other carers, with implications for their interactions with the child. Finally, maternal hostile attributions may relate to their own externalizing traits, which were not measured in the current study but may be shared by the child. Regardless of the underlying mechanisms, maternal attributions represent a potentially useful target for interventions focused on parenting behaviour and/or child aggression.

\section{Intervening role of child hostile attributions}

Our research provided limited support for the role of child hostile attributions as a key intervening process between maternal hostile attributions/negative parenting and child aggression. This was, at least in part, due to the fact that the hostile attributions-aggression association in children was modest, and was also limited to relational versus overt aggression (Crick \& Dodge, 1996; Crick et al., 2002). Stronger effects may emerge in older samples, where child attributions appear to be more stable and more closely linked to aggressive responding (Lansford et al., 2006; Orobio de Castro, Veerman, Koops, Bosch, \& Monshouwer, 2002). It is also notable that we found associations between child hostile attributions and aggression for relational but not overt aggression in the current sample. While some previous studies have also linked hostile attributions to relational aggression, associations with overt aggression have more commonly been reported in the literature (Crick \& Dodge, 1996; Mathieson et al., 2011). Relational aggression scores were higher than physical aggression scores, particularly for girls, meaning that the lack of association with overt aggression may be a consequence of relatively infrequent occurrence of these behaviours in our non-clinical sample. More generally, the existing literature examining relational/overt aggression specificity with regards to child hostile attributions is limited, and this possibility needs further empirical investigation before conclusions can be drawn. 
Regardless of the possible explanations for the limited role of child hostile attributions in the current study, parental influences on child aggression are likely to include numerous, other intervening processes. Previous research has identified numerous developmental processes that may be influenced by parenting behaviour and contribute to child externalising problems, including empathy and moral reasoning (Arsenio \& Gold, 2006), inhibitory control (Hughes \& Ensor, 2006), and emotion regulation capacities (Halligan et al., 2013). The current findings should be considered in the light of this evidence for the multifaceted role of parenting behaviour.

\section{Associations between psychosocial adversity and maternal hostile attribution tendencies}

We found robust associations between psychosocial adversity and maternal hostile attribution tendencies. Compared to their control group counterparts, mothers in the high risk group showed higher levels of child related hostile attributions at 18-months and of general hostile attributions at 5-years. This link between social context and maternal attributional style is consistent with findings that, in older children and adolescents, youth hostile attributions are inversely associated with family SES (Chen \& Matthews, 2001). Extending these observations, the current research demonstrated that parents in high risk contexts also show elevated levels of hostile attributions, including in relation to their child. High risk families in the current study were experiencing numerous adversities, including generally higher exposure to negative behaviours (e.g., criminality) in their local communities and elevated rates of specific adverse life events. The presence of higher levels of maternal hostile attributions in this context is consistent with the assumption that individuals develop social schemas based on prior experiences. However, the opposite effect, whereby maladaptive maternal attributional style is a contributor to their adverse social circumstances, cannot be ruled out. Children did not show similar risk status effects in the current sample, but at 5-years may have limited awareness of the broader social context; contextual influences may become apparent later in development. Notably, the inclusion of psychosocial adversity as a predictor in our models appeared to impact some key associations. In particular, the pathway between maternal hostile attributions and negative parenting at 18-months 
was negligible once psychosocial adversity was taken into account, and stability in maternal hostile attributions from 18-months to 5-years was also essentially eliminated. Our observations highlight the need to take account of social context in future work.

\section{Conclusions}

Strengths of the current research include the longitudinal design, the use of direct observations of maternal behaviour, and the independent measurement of key attributional and behavioural outcomes. Our results suggest that negative parenting behaviours play an intervening role in the association between maternal hostile attribution tendencies child aggression. We found less support for the role of child hostile attributions as an intervening variable, primarily due to modest correlations with child aggression. Other social influences on the development of child hostile attributions should be given further consideration. For example, more extreme adverse parental behaviours, such as physical abuse, may be particularly important to child hostile attribution tendencies (Dodge, Bates, \& Pettit, 1990); and aspects of peer relationships have also been found to be relevant, including exposure to peer victimisation (Schwartz et al., 1998) or to peer hostile attribution tendencies (Freeman, Hadwin, \& Halligan, 2011; Halligan \& Philips, 2010). Our findings should also be considered in the light of several limitations. The modest sample size limited our power to detect small effects; and although our sample was representative of the population from which it was drawn, the number of participants from minority groups was small and findings may not generalise to other samples. In addition, changes in assessments over time occurred as a consequence of the developmental approach, and the correlational design necessitates caution in drawing conclusions about causal processes. Nonetheless, overall, the results support a focus on reducing maternal hostile attributional tendencies as a potentially useful adjunct to treatment interventions aimed at altering negative parenting behaviours and/or reducing child aggression. Such interventions may be particularly relevant in high-risk populations where maternal hostile attributions are somewhat more likely to occur. 
Table 1

Descriptive statistics, reported by child gender

Male Female

\section{8-months}

Child problem behaviours (BSQ)

$1.33(0.86) \quad 1.18(0.72)$

Maternal hostile attributions present (PCCI: \%)

$27.5 \%$

$38.9 \%$

Maternal insensitivity (parenting observation:1-5)

$2.82(0.84)$

$2.43(0.59)$

5-years

Child hostile attribution score (0-1)

$0.35(0.25) \quad 0.36(0.24)$

Teacher reported total aggression (SBS: 1-5)

$2.93(1.16) \quad 2.44(0.54)$

Relational aggression subscale (SBS: 1-5)

$1.50(0.62) \quad 1.36(0.40)$

Physical aggression subscale (SBS: 1-5)

$1.43(0.68)$

$1.11(0.24)$

Maternal general hostile attributions (0-4)

$0.90(0.64) \quad 0.72(0.57)$

Maternal child-specific hostile attributions (0-4)

$1.15(0.64) \quad 1.20(0.64)$

Maternal negative control (parenting observation)

$.131(.697)$

$-.13(.703)$

BSQ: Behaviour Screening Questionnaire

SBS: Social Behavior Scale

PCCI: Parental Constraints and Concerns Interview 
Table 2: Correlation matrix

1.

2.3

4.

5.

6.

7.

8.

9.

10.

11.

1. Child gender

2. Risk status

$-.01-$

18-month variables

3. Child problem behaviours (BSQ)

$-.09 \quad .33 * * *-$

4. Maternal insensitivity

5. Maternal hostile attributions

$-.26^{*} \quad .44 * * * \quad .20 * \quad-$

$\begin{array}{llll}-.12 & .37 * * * & .36^{* * *} & .19^{+}\end{array}$

5-year child variables

6. Hostile attributions

$\begin{array}{llllll}<.01 & .05 & -.09 & -.03 & .27 * * & -\end{array}$

7. Total aggression (SBS)

8. Relational aggression (SBS)

9. Physical aggression (SBS)

$\begin{array}{llllll}-.27 * & .17 & .31 * * & .37 * * * & .08 & .14\end{array}$

$\begin{array}{llllllll}-.14 & .14 & .23 * & .21^{+} & .06 & .22 * & .88 * * *\end{array}$

$\begin{array}{llllllll}-.32 * * & .20^{+} & .31^{* *} & .43 * * * & .12 & .04 & .86 * * * & .54 * * *\end{array}$

$\underline{\text { 5-year maternal variables }}$

10. General hostile attributions (HAQ)

$\begin{array}{lllllllll}-.16 & .42 * * * & .17 & -.06 & .20 * & .18^{+} & .32 * * & .32 * * & .26 *\end{array}$

$\begin{array}{lllllllllllllll}\text { 11. Child specific hostile attributions (HAQ) } & .04 & -.02 & .08 & .13 & .15 & .10 & .14 & .13 & .10 & .29 * * & -\end{array}$

12. Negative control

$\begin{array}{lllllllllll}-.19^{+} & .53 * * * & .10 & .43 * * * & .16 & .21 * & .31 * * & .28 * & .35 * * & .39 * * * & .14\end{array}$

${ }^{+} P<.10,{ }^{*} P<.05,{ }^{* *} P<.01,{ }^{* * *} P<.001:$ BSQ: Behaviour Screening Questionnaire; SBS: Social Behavior Scale; HAQ: Hostile Attribution

Questionnaire

URL: http://mc.manuscriptcentral.com/jccap Email: mitch.prinstein@unc.edu 
Figure 1

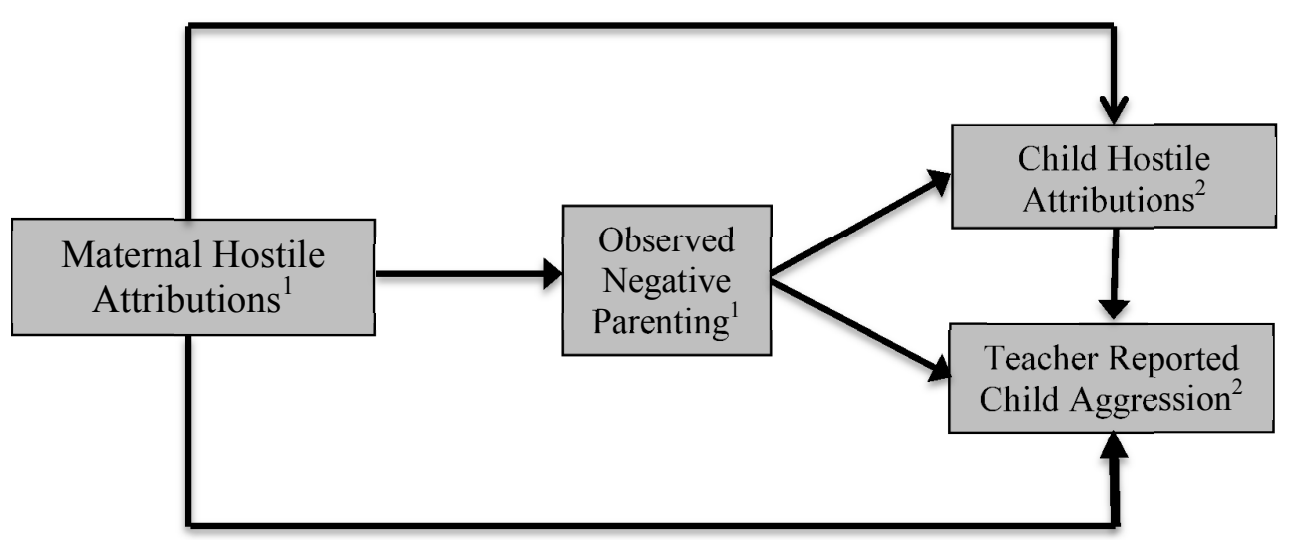

20

21

22

23

24

25

26

27

28

29

30

31

32

33

34

35

36

37

38

39

40

41

42

43

44

45

46

47

48

49

50

51

52

53

54

55

56

57

58

59

60

URL: http://mc.manuscriptcentral.com/jccap Email: mitch.prinstein@unc.edu 
Figure 2

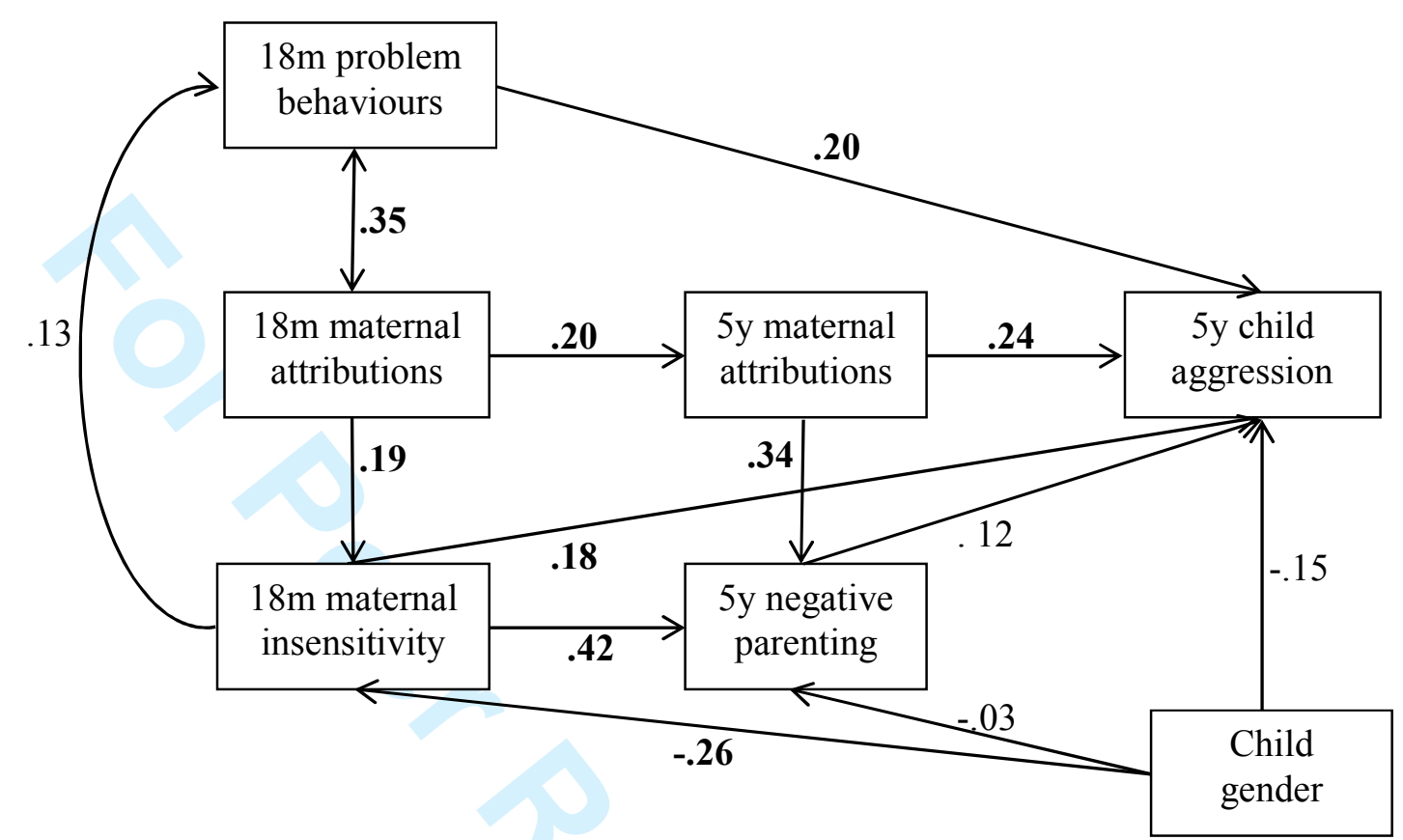

30

31

32

33

34

35

36

37

38

39

40

41

42

43

44

45

46

47

48

49

50

51

52

53

54

55

56

57

58

59

60

URL: http://mc.manuscriptcentral.com/jccap Email: mitch.prinstein@unc.edu 
Figure 3

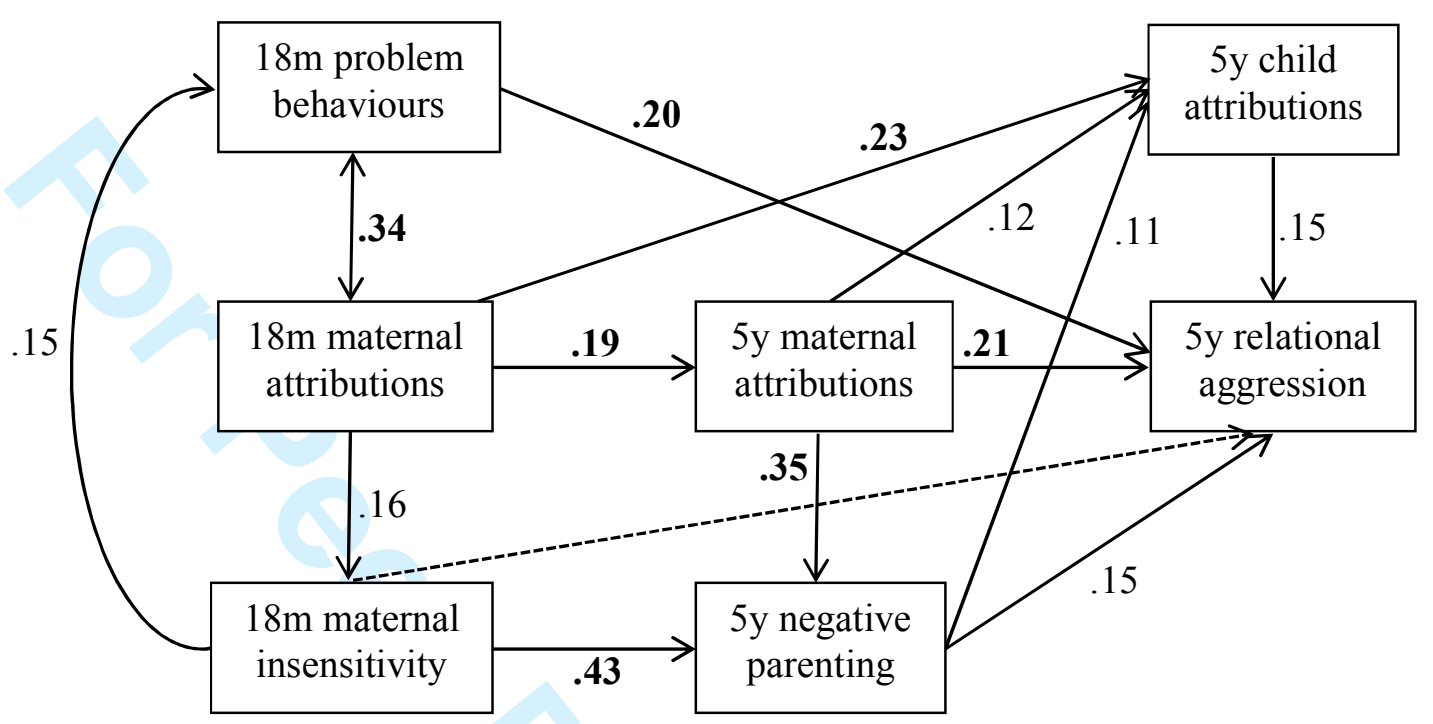


Figure 1 Title

Depiction of hypothesised associations

Figure 1 Legend

${ }^{1}$ Assessed at 18-months and 5-years child age

${ }^{2}$ Assessed at 5-years child age

Figure 2

Model examining the prediction of total child aggression, including gender effects: dashed lines indicate predicted associations that were not supported in preliminary analyses

Figure 2 footnote

Standardised coefficients are presented; paths with $P<.10$ are in bold

Figure 3

Model examining the prediction child relational aggression: dashed line indicates a weak pathway excluded from the final model

Figure 3 footnote

Standardised coefficients are presented; paths with $P<.10$ are in bold 


\section{Reference List}

Allison, P. D. (2003). Missing data techniques for structural equation modeling. Journal of Abnormal Psychology, 112, 545-557.

Arsenio, W. F. \& Gold, J. (2006). The effects of social injustice and inequality on children's moral judgments and behavior: Towards a theoretical model. Cognitive Development, 21, 388-400.

Bickett, L. R., Milich, R., \& Brown, R. T. (1996). Attributional styles of aggressive boys and their mothers. Journal of Abnormal Child Psychology, 24, 457-472.

Chen, E., Langer, D. A., Raphaelson, Y. E., \& Matthews, K. A. (2004). Socioeconomic status and health in adolescents: the role of stress interpretations. Child Development, 75, 1039-1052.

Chen, E. \& Matthews, K. A. (2001). Cognitive Appraisal Biases: An Approach to Understanding the Relation Between Socioeconomic Status and Cardiovascular Reactivity in Children. Annals of Behavioral Medicine, 23, 101-111.

Crick, N. R., Casas, J. F., \& Mosher, M. (1997). Relational and overt aggression in preschool. Developmental Psychology, 33, 579-588.

Crick, N. R. \& Dodge, K. A. (1996). Social information-processing mechanisms in reactive and proactive aggression. Child Development, 67, 993-1002.

Crick, N. R., Grotpeter, J. K., \& Bigbee, M. A. (2002). Relationally and physically aggressive children's intent attributions and feelings of distress for relational and instrumental peer provocations. Child Development, 73, 1134-1142.

Cronin, A., Halligan, S. L., \& Murray, L. (2008). Maternal psychosocial adversity and the longitudinal development of infant sleep. Infancy, 13, 469-495.

Dadds, M. R., Mullins, M. J., McAllister, R. A., \& Atkinson, E. (2003). Attributions, affect, and behavior in abuse-risk mothers: a laboratory study. Child Abuse and Neglect, 27, 21-45.

Dodge, K. A. (1980). Social cogniton and children's aggressive responses. Child Development, 51, 162-170. 
Dodge, K. A. (1986). A social information processing model of social competence in children. In M.Perlmutter (Ed.), Minnesota Symposium on Child Psychology (pp. 77-125). Hillsdale, NJ: Erlbaum.

Dodge, K. A., Bates, J. E., \& Pettit, G. S. (1990). Mechanisms in the cycle of violence. Science, 250, 1678-1683.

Dodge, K. A., Pettit, G. S., Bates, J. E., \& Valente, E. (1995). Social information-processing patterns partially mediate the effect of early physical abuse on later conduct problems. Journal of Abnormal Psychology, 104, 632-643.

Fivush, R. \& Nelson, K. (2004). Culture and language in the emergence of autobiographical memory. Psychological Science, 15, 573-577.

Freeman, K., Hadwin, J. A., \& Halligan, S. L. An experimental investigation of peer influences on hostile attributions in adolescents. Journal of Clinical Child and Adolescent Psychology, (in press).

Gardner, F. E., Sonuga-Barke, E. J., \& Sayal, K. (1999). Parents anticipating misbehaviour: an observational study of strategies parents use to prevent conflict with behaviour problem children. Journal of Child Psychology and Psychiatry, 40, 1185-1196.

Ghodsian, M., Zajicek, E., \& Wolkind, S. (1984). A Longitudinal-Study of Maternal Depression and Child-Behavior Problems. Journal of Child Psychology and Psychiatry, 25, 91-109.

Ghodsian, M., Zajicek, E., \& Wolkind, S. (1985). Comparative study of social and family correlates of children's behaviour ratings. Child: Care, Health and Development, 11, 209-228.

Gomez, R., Gomez, A., DeMello, L., \& Tallent, R. (2001). Perceived maternal control and support: effects on hostile biased social information processing and aggression among clinicreferred children with high aggression. Journal of Child Psychology and Psychiatry, 42, 513-522.

Gunning, M., Halligan, S. L., \& Murray, L. (2013). Contributions of maternal and infant factors to infant responding to the Still Face paradigm: A longitudinal study. Infant Behav.Dev, 36, $319-328$. 
Halligan, S. L., Cooper, P. J., Fearon, R. M. P., Sampson, S., Crosby, M., \& Murray, L. (2013). The longitudinal development of emotion regulation capacities in children at risk for externalizing disorders. Development and Psychopathology, 25, 391-406.

Halligan, S. L., Cooper, P. J., Healy, S. J., \& Murray, L. (2007). The attribution of hostile intent in mothers, fathers and their children. Journal of Abnormal Child Psychology, 35, 594-604.

Halligan, S. L. \& Philips, K. J. (2010). Are you thinking what I'm thinking? Peer group similarities in adolescent hostile attribution tendencies. Developmental Psychology, 46, 1385-1388.

Hughes, C. \& Ensor, R. (2006). Behavioural problems in 2-year-olds: links with individual differences in theory of mind, executive function and harsh parenting. Journal of Child Psychology and Psychiatry, 47, 488-497.

Juliano, M., Werner, R., \& Wright Cassidy, K. (2006). Early correlates of prechool aggressive behaviour according to type of aggression and measurement. Journal of Applied Developmental Psychology, 27, 395-410.

Lansford, J. E., Malone, P. S., Dodge, K. A., Crozier, J. C., Pettit, G. S., \& Bates, J. E. (2006). A 12-year prospective study of patterns of social information processing problems and externalizing behaviors. Journal of Abnormal Child Psychology, 34, 715-724.

MacBrayer, E. K., Milich, R., \& Hundley, M. (2003). Attributional biases in aggressive children and their mothers. Journal of Abnormal Psychology, 112, 698-708.

MacKinnon-Lewis, C., Lamb, M. E., Arbuckle, B., Baradaran, L. P., \& Volling, B. L. (1992). The relationship between biased maternal and filial attributions and the aggressiveness of their interactions. Development and Psychopathology, 4, 403-415.

MacKinnon-Lewis, C., Lamb, M. E., Hattie, J., \& Baradaran, L. P. (1999). A longitudinal examination of the associations between mothers' and sons' attributions and their aggression. Development and Psychopathology, 13, 69-81.

Mathieson, L. C., Murray-Close, D., Crick, N. R., Woods, K. E., Zimmer-Gembeck, M., Geiger, T. C. et al. (2011). Hostile intent attributions and relational aggression: the moderating roles of emotional sensitivity, gender, and victimization. J Abnorm.Child Psychol., 39, 977-987. 
Murray, L. (1992). The impact of postnatal depression on infant development. Journal of Child Psychology and Psychiatry, 33, 543-561.

Murray, L., FioriCowley, A., Hooper, R., \& Cooper, P. (1996). The impact of postnatal depression and associated adversity on early mother-infant interactions and later infant outcome. Child Development, 67, 2512-2526.

Nelson, D. A. \& Coyne, S. M. (2009). Children's intent attributions and feelings of distress: associations with maternal and paternal parenting practices. Journal of Abnormal Child Psychology, $37,223-237$.

Nelson, D. A., Mitchell, C., \& Yang, C. (2008). Intent attributions and aggression: a study of children and their parents. Journal of Abnormal Child Psychology, 36, 793-806.

Nix, R. L., Pinderhughes, E. E., Dodge, K. A., Bates, J. E., Pettit, G. S., \& McFadyenKetchum, S. A. (1999). The relation between mothers' hostile attribution tendencies and children's externalizing behavior problems: the mediating role of mothers' harsh discipline practices. Child Development, 70, 896-909.

O'Connor, T. G. (2002). Annotation: The 'effects' of parenting reconsidered: findings, challenges, and applications. J Child Psychol Psychiatry, 43, 555-572.

Orobio de Castro, B., Veerman, J. W., Koops, W., Bosch, J. D., \& Monshouwer, H. J. (2002). Hostile attribution of intent and aggressive behavior: a meta-analysis. Child Development, 73, 916934.

Pettit, G. S., Bates, J. E., \& Dodge, K. A. (1997). Supportive parenting, ecological context, and children's adjustment: A seven-year longitudinal study. Child Development, 68, 908-923.

Pettit, G. S., Laird, R. D., Bates, J. E., Dodge, K. A., \& Criss, M. M. (2001). Antecedents and behavior-problem outcomes of parental monitoring and psychological control in early adolescence. Child Development, 72, 583-598.

Richman, N. \& Graham, P. J. (1971). A Behavioural Screening Questionnaire for use with three-year-old children: preliminary findings. Journal of Child Psychology and Psychiatry, 12, 533. 
Rutter, M., Giller, H., \& Hagell, A. (1998). Antisocial Behavior by Young People. Cambridge, England: Cambridge University Press.

Schwartz, D., Dodge, K. A., Coie, J. D., Hubbard, J. A., Cillessen, A. H., Lemerise, E. A. et al. (1998). Social-cognitive and behavioral correlates of aggression and victimization in boys' play groups. Journal of Abnormal Child Psychology, 26, 431-440.

Shaw, D. S., Winslow, E. B., Owens, E. B., \& Hood, N. (1998). Young children's adjustment to chronic family adversity: a longitudinal study of low-income families. Journal of the American Academy of Child and Adolescent Psychiatry, 37, 545-553.

Sheridan, A., Murray, L., Cooper, P. J., Evangeli, M., Byram, V., \& Halligan, S. L. (2013). A longitudinal study of child sleep in high and low risk families: relationship to early maternal settling strategies and child psychological functioning. Sleep Medicine, 14, 266-273.

Snyder, J., Cramer, A., Afrank, J., \& Patterson, G. R. (2005). The contributions of ineffective discipline and parental hostile attributions of child misbehavior to the development of conduct problems at home and school. Developmental Psychology, 41, 30-41.

Weiss, B., Dodge, K. A., Bates, J. E., \& Pettit, G. S. (1992). Some consequences of early harsh discipline: child aggression and a maladaptive social information processing style. Child Development, 63, 1321-1335.

Wolke, D., Skuse, D., \& Mathisen, B. (1990). Behavioral style in failure-to-thrive infants: a preliminary communication. Journal of Pediatric Psychology, 15, 237-254.

Zaslow, M. J., Weinfield, N. S., Gallagher, M., Hair, E. C., Ogawa, J. R., Egeland, B. et al. (2006). Longitudinal prediction of child outcomes from differing measures of parenting in a lowincome sample. Developmental Psychology, 42, 27-37. 